\title{
Carcass Composition and Quality of Meat of Pulawska and Pulawska x PLW Crossbred Pigs Fed Rations with Naked Oats
}

\author{
Anna Milczarek \\ Institute of Animal Science and Fisheries, Faculty of Agrobioengineering and Animal Husbandry, Siedlce \\ University of Natural Sciences and Humanities, Bolesława Prusa 14, 08-110 Siedlce, Poland; \\ anna.milczarek@uph.edu.pl; Tel.: +48-25-643-13-77
}

check for

updates

Citation: Milczarek, A. Carcass Composition and Quality of Meat of Pulawska and Pulawska x PLW Crossbred Pigs Fed Rations with Naked Oats. Animals 2021, 11, 3342. https://doi.org/10.3390/ ani11123342

Academic Editors: Eugeniusz Ryszard Grela and Małgorzata Swiątkiewicz

Received: 8 October 2021

Accepted: 21 November 2021

Published: 23 November 2021

Publisher's Note: MDPI stays neutral with regard to jurisdictional claims in published maps and institutional affiliations.

Copyright: (C) 2021 by the author. Licensee MDPI, Basel, Switzerland. This article is an open access article distributed under the terms and conditions of the Creative Commons Attribution (CC BY) license (https:/ / creativecommons.org/licenses/by/ $4.0 /)$.
Simple Summary: Due to the presence of fibrous hulls, the use of traditional cultivars of oats (Avena sativa L.) in feed rations for pigs is restricted. The development of naked oat cultivars (Avena nuda L.) containing only about $2 \%$ of crude fibre allowed this cereal grain to be included in the diets of monogastric animals. The present studies evaluated the efficiency of a $60 \%$ inclusion of naked oats in rations fed to Pulawska pigs and Pulawska $x$ Polish Large White (PLW) crossbreds on their performance results (weight gain, feed conversion), carcass composition and physical ( $\mathrm{pH}$, colour, WHC) and chemical (essential nutrients, fatty acids) characteristics of meat. It was demonstrated that a $60 \%$ inclusion of naked oats is recommended in the diets of Pulawska pigs and Pulawska x PLW crossbreds to maintain carcass composition and meat quality comparable to that of pigs fed with rations containing barley only. The performance indicators of Pulawska pigs declined, but their meat quality traits improved in terms of nutritional value, as the n-6/n-3 acids' ratio was narrower than that of Pulawska x PLW.

Abstract: The study aimed to determine the impact of a $60 \%$ inclusion of naked oats in feed rations for Pulawska pigs and Pulawska $x$ Polish Large White (PLW) crossbreds on the fattening performance, carcass composition, and meat quality. It was demonstrated that-independent from their dietPulawska pigs showed a daily weight gain about $14.5 \%$ lower $(p \leq 0.05)$, with about $15 \%$ higher feed conversion rate. The experimental factors showed no impact on the dressing percentage, meatiness, and backfat thickness, except that the "eye" of the loin was significantly smaller (by $4.55 \mathrm{~cm}^{2}$ ) in Pulawska pigs. There was no interaction effect (diet/breed) for the pigs' performance results and carcass composition. A $60 \%$ inclusion of naked oats in the pigs' diet did not affect the weight of primal cuts in the right-side half-carcass. Compared to Pulawska pigs, crossbreds featured a significantly lower weight of bacon and ribs (by $1.17 \mathrm{~kg}$ ) but a higher $(p \leq 0.05)$ weight of fillet (by $1.0 \mathrm{~kg}$ ) and ham with shin (by $0.43 \mathrm{~kg}$ ). The diet had no impact on the evaluated muscles, except a reduction (by 2.3 points) in colour lightness (L) of musculus semimembranosus in fattening pigs receiving feed rations with a $60 \%$ share of oats. Both muscles in Pulawska pigs showed significantly better $(p \leq 0.05)$ water-holding capacity and reduced colour lightness (L) in comparison to crossbred pigs. Moreover, the longissimus lumborum muscle of Pulawska pigs had a higher chroma $(\mathrm{C})$ and a lower hue $(\mathrm{H})$. The contents of essential nutrients in the evaluated muscles did not depend on the pigs' diet and breed, except that a higher by $0.3 \%(p \leq 0.05)$ intramuscular fat content was found in the longissimus lumborum muscle of Pulawska pigs. Neither of the experimental factors showed significant impact on the total saturated fatty acids (SFA) and total unsaturated fatty acids (UFA), or on neutral or hypocholesterolemic (DFA) and hypercholesterolemic fatty acids (OFA) in the evaluated muscles. The muscles of Pulawska x PLW pigs contained more (by $1.77 \%$ FA in the longissimus lumborum and $1.16 \%$ in the semimembranosus) polyunsaturated fatty acids (PUFA) than the muscles of the Pulawska breed $(p \leq 0.05)$. In addition, naked oats included in the pigs' diet increased $(p \leq 0.05)$ the share of PUFA in intramuscular fat. The muscles of Pulawska pigs, in comparison to the muscles of crossbreds, showed a significantly improved ratio: by $66 \%$ and $69 \%$ in the longissimus lumborum and the semimembranosus muscles, respectively. To sum up, a $60 \%$ inclusion of naked oats is recommended in the rations of both Pulawska pigs and Pulawska x PLW crossbreds, since it allows satisfactory 
carcass composition and meat quality to be maintained. Pulawska pigs had worse productivity ratios but showed improved meat quality traits in terms of the $n-6 / n-3$ ratio.

Keywords: naked oats; performance results; carcass composition; physical-chemical properties of meat; fattening pigs

\section{Introduction}

Pork accounts for nearly $40 \%$ of the global production and consumption of meat [1]. It is a source of complete protein and significant amounts of easily assimilated iron, zinc, selenium, copper, and vitamins from the B group, as well as bioactive compounds [2-4]. The content of fat and its fatty acid profile are nutritionally essential $[5,6]$. Increased awareness makes consumers look for culinary meat of high quality, reflected in sensory traits and health properties. Most consumers prefer light pink meat with minimum fat and juice drip. A darker colour of meat and excessive drip are associated with the loss of freshness [7]. For heat-treated meat, the main consumer focus is on its sensory quality, which is dependent mostly on juice drip and fat content. All the above-mentioned characteristics affect the quality of the meat, which depend on the breed of pigs, their genotype, pigs' diet, and handling of animals before and after slaughter [3,8-10]. Pork from Pulawska pigs can be an attractive raw material for high-quality meat products. The Pulawska is a native breed from the eastern Lublin region and western Masovia in Poland. Compared with typical meat pig breeds, it features lower fattening ratios (lower daily weight gain and increased feed conversion), lower meatiness, and slightly increased fat percentage, but the meat is clearly of higher quality [2,10-13]. A significant element contributing to the high quality of pork meat from Pulawska pigs is the structure of the muscle fibres and the content of intramuscular fat (IMF), determining its palatability. As reported by Kasprzyk [11], Babicz [14], and Wojtysiak and Połtowicz [15], this creates a possibility of using Pulawska pigs for producing brand-name products, e.g., Pulawska ham. Bocian et al. [16], Debrecéni et al. [17], Matoušek et al. [18], and Colonna et al. [19] claimed that native breeds are raised for culinary meat and that the interest in products made traditionally is greater in the United States and some European countries e.g., Hungary, Switzerland, Austria, Germany, Spain, Great Britain, and Poland. Studies [9,14] have demonstrated that good-quality pork can be obtained by crossbreeding native and commercial breeds. Such crossbreds usually show improved fattening performance and carcass composition in comparison with native breeds of pigs.

Numerous studies $[8,9,20-22]$ have demonstrated that the pigs' diet is an important environmental factor affecting pork quality, including its nutritional value. The underlying components of the diets of growing pigs are cereal grains: barley, wheat, and triticale $[23,24]$. Due to the presence of fibrous hulls, the use of traditional cultivars of oat (Avena sativa L.) in feed rations for swine is restricted. The development of naked oat cultivars (Avena nuda L.) containing about $2 \%$ of crude fibre is allowed use of this cereal grain in the diets of monogastric animals $[25,26]$. The characteristic feature of protein is a good amino acid composition with a high nutritional value. Studies by Brand and Merwe [27] demonstrated that naked oat cultivars have a higher nutritional protein value than those of other cereals, although lysine is still the limiting amino acid. A high fat level is a good source of essential unsaturated fatty acids $[28,29]$. A negative feature of naked oats is the presence of considerable amounts of $\beta$-glucans-antinutrients that, according to different authors $[25,30,31]$ can account for up to $6 \%$ of dry matter. In barley, $\beta$-glucans account for $3.2-4.6 \%$ of its dry matter, in oats, $3.9-5.7 \%$, and in wheat and triticale, $0.5-1.1 \%[32,33]$. In order to reduce or completely eliminate the negative impact of these substances on animal bodies, it is necessary to use appropriately selected enzymatic preparations $[9,34]$. Numerous studies $[9,21,22,27,35]$ have shown that complete feed rations for growing pigs could contain up to $73.5 \%$ of naked oats, but their production and slaughter performance 
varied. In addition, the available literature lacks studies comparing the qualification of naked oats for the diets of slow-growing breeds of pigs and their crossbreds.

Therefore, the study aimed to determine the impact of $60 \%$ naked oat on feed rations for Pulawska pigs and Pulawska x PLW (Polish Large White) crossbreds on the fattening performance, carcass composition, and quality of meat.

\section{Materials and Methods}

\subsection{Experimental Design}

The study involved 40 pigs of the Pulawska breed (P) and 40 Pulawska x PLW crossbred pigs (M). All the animals were fattened starting from average body weight of $29.5 \mathrm{~kg}$ up to about $102 \mathrm{~kg}$. Therefore, for Pulawska x PLW crossbreds, the fattening period lasted 90 days, and for Pulawska pigs, 105 days. The pigs were kept in pens (20 animals per pen) with deep litter, equipped with automatic feed dispensers and nipple drinkers. The animals were fed ad libitum. Twenty Pulawska pigs and 20 Pulawska x PLW crossbreds received feed rations based on barley meal (J), and 20 Pulawska pigs and 20 Pulawska $x$ PLW crossbreds were diets containing $60 \%$ of naked oat meal replacing $2 / 3$ of barley $(\mathrm{O})$ (Table 1).

Table 1. Raw materials (\%) and nutritive value of diets.

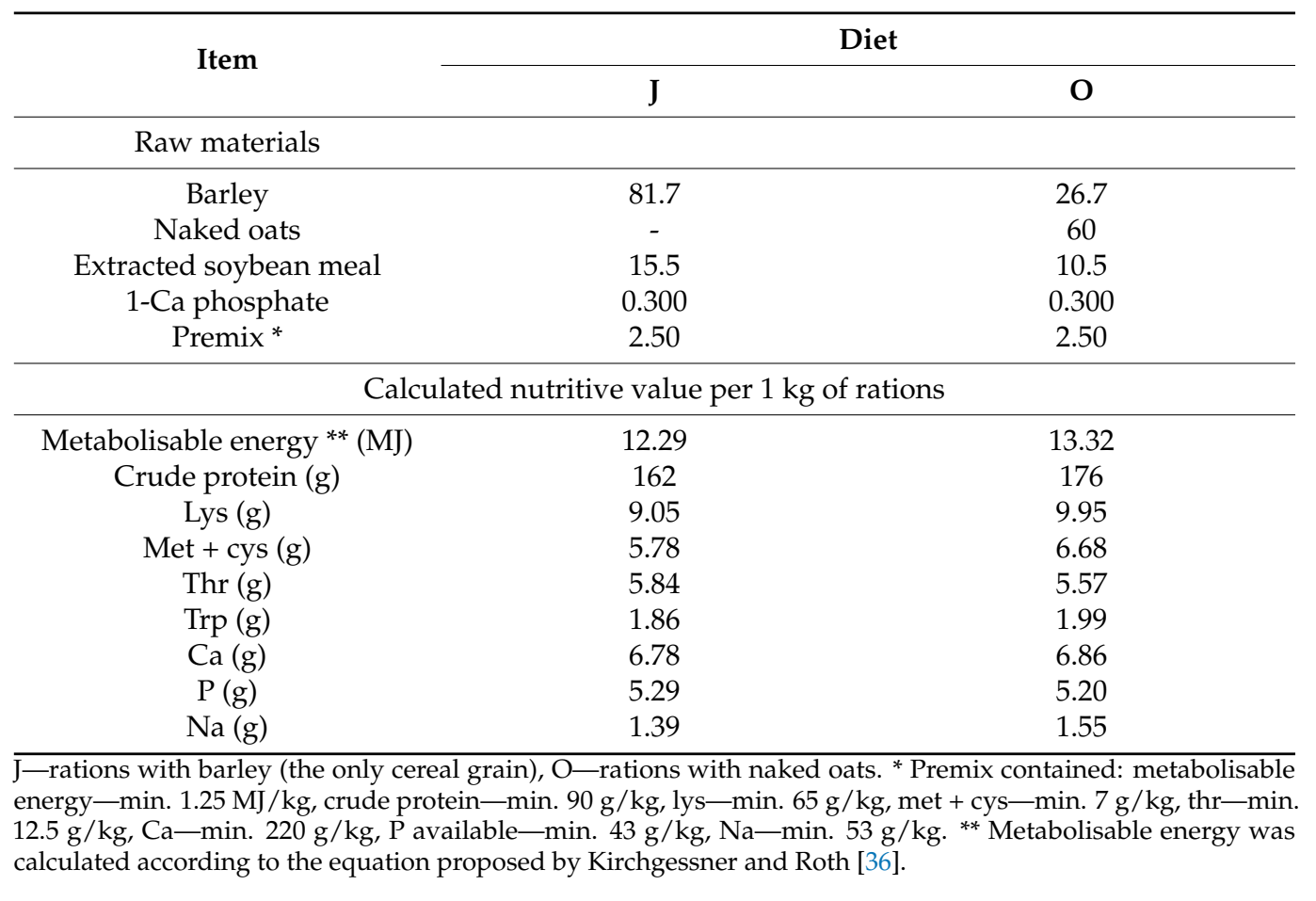

The rations were balanced in accordance with nutritional recommendations [37], assuming the content of basic components according to the current results (Table 2) of chemical analyses in raw materials, while the remaining data were based on the tables [37]. The partial replacement of barley with naked oats increased the metabolisable energy in the experimental ration by $1.12 \mathrm{MJ}$ and the lysine content by $0.9 \mathrm{~g}$. However, the content of protein and lysine per $1 \mathrm{MJ}$ ME was identical in both feed rations, while the percentage of extracted soybean meal in the experimental rations was reduced ( $\mathrm{O}$ rations). 
Table 2. Chemical composition of naked oats.

\begin{tabular}{|c|c|}
\hline Item & Contents \pm SD \\
\hline \multicolumn{2}{|c|}{ Essential nutrients (g/kg dry matter) } \\
\hline Dry matter & $885 \pm 13.2$ \\
\hline Crude ash & $23.73 \pm 0.174$ \\
\hline Crude protein & $186.44 \pm 2.53$ \\
\hline Crude fat & $57.28 \pm 1.07$ \\
\hline Crude fibre & $29.72 \pm 1.37$ \\
\hline Nitrogen-free extractives (NFE) & $587.83 \pm 16.1$ \\
\hline \multicolumn{2}{|c|}{ Amino acids (g/16 g N) } \\
\hline Lys & $4.42 \pm 0.140$ \\
\hline Met & $1.43 \pm 0.089$ \\
\hline Cys & $2.55 \pm 0.110$ \\
\hline Thr & $3.76 \pm 0.117$ \\
\hline $\operatorname{Trp}$ & $1.02 \pm 0.104$ \\
\hline \multicolumn{2}{|c|}{ Macroelements (g/kg) } \\
\hline $\mathrm{Ca}$ & $0.620 \pm 0.020$ \\
\hline $\mathrm{P}$ & $5.11 \pm 0.110$ \\
\hline $\mathrm{K}$ & $3.09 \pm 0.101$ \\
\hline $\mathrm{Na}$ & $0.108 \pm 0.013$ \\
\hline$\beta$-glucans (\% dry matter) & $4.28 \pm 0.295$ \\
\hline
\end{tabular}

Prior to and after the experiment, all the animals were weighed individually and the results were used as a reference to calculate daily weight gains. During fattening, the feed intake (FI) was strictly controlled in order to calculate the feed conversion ratio (FCR).

\subsection{Evaluation of the Pigs' Carcass Composition}

On the final day of the trial the pigs were slaughtered. In order to evaluate carcass composition and meat quality, 10 pigs with body weights representative of a specific group were selected from each group. According to the technology used by meat processing plants, the pigs were slaughtered two hours after transport $(30 \mathrm{~km})$ by electric stunning and bleeding in a horizontal position. The meatiness of the pigs was evaluated using an ULTRA-FOM 300 apparatus (SFK, Peosta, IA, USA). Afterwards, the carcasses were cooled for $24 \mathrm{~h}$ at a temperature of $0-4{ }^{\circ} \mathrm{C}$. The length of the carcass was measured on the rightside half-carcass, and backfat thickness was determined at five points: above the shoulder, mid-back (between the last thoracic vertebra and the first lumbar vertebra), and over the loin (at the level of sacral vertebrae: I, II, III). Afterwards, the right-side half-carcasses were cut using the SKURTCh method [38]. During dissection, samples of musculus longissimus lumborum and musculus semimembranosus were taken from each carcass ( $n=10$ per muscle) and set up in twos ( $n=5$ per muscle) for the evaluation of the physicochemical properties of the meat.

\subsection{Evaluation of the Chemical Composition of Naked Oats and Meat}

The dry matter, total ash, crude protein, crude fat, and crude fibre contents were described using the AOAC [39] method: dry matter (930.15), total ash (942.05), crude protein (990.03), crude fat (991.36), and crude fibre (978.10) for naked oats only. The share of nitrogen-free extractives (NFE) in naked oats was calculated using the following formula:

$$
\mathrm{NFE}=\text { dry matter }-(\text { crude protein }+ \text { total ash }+ \text { crude fat }+ \text { crude fibre }) \text {. }
$$

The content of amino acids in naked oats was determined by ion-exchange chromatography, using an AAA-T400 amino acid analyser made by Microtechna Prague with suitable hydrolysed proteins: tryptophan was determined by spectrophotometry using 
ap-dimethylaminobenzaldehyde (DAMB), methionine, and cysteine, following performic acid oxidation of the sample, and other amino acids following acid hydrolysis of protein.

Comminuted samples of oats were weighed and wet-mineralised in a mixture of $\mathrm{HNO}_{3}$ and $\mathrm{HClO}_{4}$ (at a 3:1 ratio). The mineralisation was conducted in a block made by the Tecator company with a programmed temperature. Next, in the resulting mineralisate, the contents of macroelements - calcium, sodium, and potassium - were determined by flame atomic emission spectrometry in a Flacho 4 photometer made by Carl Zeiss-Jena and that of phosphorus by colorimetry using eikonogen as a reducing agent.

The level of $\beta$-glucans in naked oats was determined via the enzymatic method of McCleary and Codd using an assay kit obtained from Megazyme International Ltd. (Bray, Ireland) [40].

Fatty acids were separated using the Folch method [41]. The fatty acid profile of the lipid fraction was determined by gas chromatography (GC) of methyl esters using a Varian 450-GC gas chromatograph with a flame ionisation detector (air-hydrogen). A Select ${ }^{\mathrm{TM}}$ Biodiesel for FAME capillary column was used $(30 \mathrm{~m}, 0.32 \mathrm{~mm}, 0.25 \mu \mathrm{m})$ with a Select Biodiesel for FAME Fused Silica filling. The injector temperature was $250{ }^{\circ} \mathrm{C}$, detector temperature $300^{\circ} \mathrm{C}$, and column temperature $200^{\circ} \mathrm{C}$ (initial) and $240{ }^{\circ} \mathrm{C}$ (final). Helium was used as a carrier gas, with a flow of $2.5 \mathrm{~mL}$ per minute. The neutral or hypocholesterolemic fatty acids (DFA) and hypercholesterolemic fatty acids (OFA) were calculated based on the percentage (\%) of total fatty acids [29], using the following formulas:

$$
\text { DFA = MUFA (monounsaturated fatty acids) + C18:0, OFA = C14:0 + C16:0. }
$$

\subsection{Evaluation of the Physical Properties of Muscles}

The concentration of hydrogen ions $\left(\mathrm{pH}_{45}\right.$ and $\left.\mathrm{pH}_{24}\right)$ in longissimus muscles was measured using a Testo $205 \mathrm{pH}$ meter with a dagger electrode. Forty-five minutes after the slaughter and after more than $24 \mathrm{~h}$ of cooling, muscle reaction $\left(\mathrm{pH}_{45}\right.$ and $\left.\mathrm{pH}_{24}\right)$ was measured.

Water losses, expressed as water-holding capacity (WHC), were determined using Grau and Hamm's [42] method as modified by Pohja and Ninivarra [43], based on how much free water (expressed in \%) is lost by the sample of meat placed on the filter paper pressed between two glass plates. The infiltration area $\left(\mathrm{cm}^{2}\right)$ was measured using a planimeter and calculated as the content of free water, assuming that $1 \mathrm{~cm}^{2}$ of filter paper absorbs $10 \mathrm{mg}$ of muscle juice.

According to the $\mathrm{L}, \mathrm{a}^{*}, \mathrm{~b}^{*}$ system, the colour of longissimus lumborum and semimembranosus muscles was determined using a Minolta Chroma Meter CR 300 (Konica Minolta, Osaka, Japan) [44]. Two illuminant/observer combinations were applied, i.e., illuminant $\mathrm{C}$ (average day light) and standard observer $2^{\circ}$, as well as illuminant D65 (day light) and standard observer $10^{\circ}$, recommended for measurements of meat colour [45]. In the measuring system used, $\mathrm{L}$ denotes psychometric colour saturation which is a spatial vector. On the other hand, $\mathrm{a}^{*}$ and $\mathrm{b}^{*}$ are trichromatic coordinates, where $\mathrm{a}^{*}$ being a positive value corresponds to red, and being a negative value to green; in turn, positive $b^{*}$ corresponds to yellow and negative $b^{*}$ to blue. The colour parameters $a^{*}$ and $b^{*}$ were used to calculate chroma (C) and the proportion of redness to yellowness (H; hue) [46] with the following formulas:

$$
\mathrm{C}=\left[\left(\mathrm{a}^{*}\right)^{2}+\left(\mathrm{b}^{*}\right)^{2}\right]^{0.5}, \mathrm{H}=\log \left(\mathrm{b}^{*} / \mathrm{a}^{*}\right) .
$$

\subsection{Statistical Analysis}

The results were elaborated by statistical methods using a two-way analysis of variance. The significance of differences between mean values was verified at the significance level $\alpha \leq 0.05$. The obtained data were tested using the post hoc Tukey's test. The results were processed with STATISTICA PL 13.1 software [47]. 


\section{Results}

A $60 \%$ inclusion of naked oats in diets had no significant $(p>0.05)$ influence on the pigs' weight gain (Table 3$)$. Independent from the pigs' diet, a significantly lower $(p \leq 0.05)$ daily weight gain by about $14.5 \%$, and higher feed conversion ratio by about $15 \%$ were recorded in Pulawska pigs compared to Pulawska x PLW crossbreds.

Table 3. Fattening performance and carcass composition.

\begin{tabular}{|c|c|c|c|c|c|c|c|c|}
\hline \multirow{2}{*}{ Item } & \multirow{2}{*}{ Breed (B) } & \multicolumn{2}{|c|}{ Diet (F) } & \multirow[t]{2}{*}{$\overline{\mathbf{x}}$} & \multicolumn{3}{|c|}{$p$-Value } & \multirow{2}{*}{ SEM } \\
\hline & & $\mathbf{J}$ & $\mathrm{O}$ & & $\mathbf{F}$ & B & F/B & \\
\hline \multirow{4}{*}{$\begin{array}{l}\text { Body weight }(\mathrm{kg}) \\
\quad \text { - initial }\end{array}$} & $\mathrm{P}$ & 29.8 & 29.7 & 29.75 & & & & \\
\hline & $\mathrm{M}$ & 29.6 & 29.2 & 29.40 & 0.501 & 0.436 & 0.492 & 0.975 \\
\hline & $\overline{\mathrm{x}}$ & 29.70 & 29.45 & & & & & \\
\hline & $\mathrm{P}$ & 102.7 & 102.1 & 102.40 & & & & \\
\hline \multirow[t]{2}{*}{ - final } & M & 102.3 & 102.3 & 102.30 & 0.534 & 0.582 & 0.615 & 0.888 \\
\hline & $\bar{x}$ & 102.50 & 102.20 & & & & & \\
\hline \multirow{4}{*}{$\begin{array}{l}\text { Daily weight gain } \\
\text { (g) }\end{array}$} & $\mathrm{P}$ & 694 & 690 & $692^{b}$ & & & & \\
\hline & $\mathrm{M}$ & 808 & 813 & $810^{a}$ & 0.724 & $<0.05$ & 0.053 & 0.682 \\
\hline & $\overline{\mathrm{x}}$ & 751 & 751 & & & & & \\
\hline & $\mathrm{P}$ & 3.10 & 3.24 & 3.17 & & & & \\
\hline \multirow[t]{2}{*}{ FCR $(\mathrm{kg})$} & M & 2.74 & 2.77 & 2.76 & - & - & - & - \\
\hline & $\bar{x}$ & 2.92 & 3.01 & & & & & \\
\hline \multirow{4}{*}{$\begin{array}{c}\text { Dressing } \\
\text { percentage }(\%)\end{array}$} & $\mathrm{P}$ & 76.3 & 77.4 & 76.9 & & & & \\
\hline & M & 76.9 & 77.1 & 77.0 & 0.343 & 0.681 & 0.058 & 0.290 \\
\hline & $\bar{x}$ & 76.6 & 77.3 & & & & & \\
\hline & $\mathrm{P}$ & 51.2 & 50.0 & 50.6 & & & & \\
\hline \multirow[t]{2}{*}{ Meatiness (\%) } & M & 53.0 & 53.0 & 53.0 & 0.597 & 0.049 & 0.221 & 0.467 \\
\hline & $\overline{\mathrm{x}}$ & 52.1 & 51.5 & & & & & \\
\hline \multirow{4}{*}{$\begin{array}{c}\text { Loin "eye" area } \\
\left(\mathrm{cm}^{2}\right)\end{array}$} & $\mathrm{P}$ & 45.5 & 45.0 & $45.3^{b}$ & & & & \\
\hline & $\mathrm{M}$ & 50.0 & 49.6 & $49.8^{a}$ & 0.856 & $<0.05$ & 0.968 & 1.07 \\
\hline & $\bar{x}$ & 47.8 & 47.3 & & & & & \\
\hline & $\mathrm{P}$ & 1.68 & 1.76 & 1.72 & & & & \\
\hline \multirow[t]{2}{*}{ Lard weight (kg) } & M & 1.55 & 1.44 & 1.50 & 0.323 & 0.134 & 0.319 & 0.063 \\
\hline & $\bar{x}$ & 1.62 & 1.60 & & & & & \\
\hline \multirow{3}{*}{$\begin{array}{l}\text { Carcass length } \\
(\mathrm{cm})\end{array}$} & $\mathrm{P}$ & 80.4 & 79.8 & 80.1 & & & & \\
\hline & M & 80.5 & 80.4 & 80.5 & 0.901 & 0.483 & 0.205 & 0.297 \\
\hline & $\bar{x}$ & 80.5 & 80.1 & & & & & \\
\hline \multirow{2}{*}{$\begin{array}{l}\text { Backfat thickness } \\
(\mathrm{mm})\end{array}$} & $\mathrm{P}$ & 37.33 & 42.7 & 40.02 & & & & \\
\hline & M & 37.43 & 37.1 & 37.27 & 0.305 & 0.163 & 0.156 & 1.00 \\
\hline \multirow[t]{2}{*}{ - over the shoulder } & $\bar{x}$ & 37.38 & 39.90 & & & & & \\
\hline & $\mathrm{P}$ & 19.78 & 25.6 & 22.69 & & & & \\
\hline \multirow[t]{3}{*}{ - mid back } & M & 22.78 & 23.00 & 22.89 & 0.053 & 0.873 & 0.055 & 0.692 \\
\hline & $\overline{\mathrm{x}}$ & 21.28 & 24.30 & & & & & \\
\hline & $\mathrm{P}$ & 28.11 & 29.50 & 28.81 & & & & \\
\hline \multirow[t]{3}{*}{ - sacrum I } & M & 26.51 & 25.20 & 25.86 & 0.932 & 0.126 & 0.566 & 0.901 \\
\hline & $\bar{x}$ & 27.31 & 27.35 & & & & & \\
\hline & $\mathrm{P}$ & 18.78 & 22.00 & 20.39 & & & & \\
\hline \multirow[t]{3}{*}{ - sacrum II } & M & 18.58 & 19.2 & 18.89 & 0.275 & 0.678 & 0.753 & 0.876 \\
\hline & $\bar{x}$ & 18.68 & 20.60 & & & & & \\
\hline & $\mathrm{P}$ & 31.00 & 34.10 & 32.55 & & & & \\
\hline \multirow[t]{2}{*}{ - sacrum III } & M & 29.60 & 26.10 & 27.85 & 0.955 & 0.103 & 0.291 & 1.35 \\
\hline & $\overline{\mathrm{x}}$ & 30.30 & 30.10 & & & & & \\
\hline - arithmetic mean & $\mathrm{P}$ & 27.00 & 30.36 & 28.68 & & & & \\
\hline from 5 & M & 26.98 & 26.12 & 26.55 & 0.448 & 0.179 & 0.189 & 0.818 \\
\hline measurements & $\bar{x}$ & 26.99 & 28.24 & & & & & \\
\hline
\end{tabular}

J-rations with barley (the only cereal grain), O—rations with naked oats, FCR—feed conversion ratio, B-breed, F-diet, SEM-standard error of the mean, $n=20$ for fattening performance, $n=10$ for carcass composition traits, ab-means with different superscripts are significantly different at $p \leq 0.05$. 
When analysing the carcass composition of pigs, the pigs' diet and breed were not observed to have an impact on the pigs' dressing percentage meatiness and backfat thickness, except that the "eye" of the loin was significantly smaller (by 9\%) in Pulawska pigs compared to crossbreds of this breed with PLW pigs. No interaction effect of experimental factors $(\mathrm{F} / \mathrm{B})$ was observed in the study for the pigs' performance results and carcass composition.

The rations fed to Pulawska pigs and Pulawska $x$ PLW crossbreds did not affect the weights of primal cuts in the right-side half-carcass (Table 4).

Table 4. Primal cuts weight $(\mathrm{kg})$ of right half-carcass.

\begin{tabular}{|c|c|c|c|c|c|c|c|c|c|}
\hline \multirow{2}{*}{\multicolumn{2}{|c|}{ Item }} & \multirow{2}{*}{ Breed (B) } & \multicolumn{2}{|c|}{ Diet (F) } & \multirow[t]{2}{*}{$\overline{\mathbf{x}}$} & \multicolumn{3}{|c|}{$p$-Value } & \multirow{2}{*}{ SEM } \\
\hline & & & $\mathrm{J}$ & O & & F & B & F/B & \\
\hline \multirow{4}{*}{\multicolumn{2}{|c|}{ neck }} & $\mathrm{P}$ & 5.84 & 6.20 & 6.02 & & & & \\
\hline & & $\mathrm{M}$ & 6.53 & 6.42 & 6.48 & 0.351 & 0.077 & 0.499 & 0.114 \\
\hline & & $\bar{x}$ & 6.19 & 6.31 & & & & & \\
\hline & & $\mathrm{P}$ & 4.32 & 4.15 & 4.24 & & & & \\
\hline \multirow{3}{*}{\multicolumn{2}{|c|}{ shoulder }} & M & 3.98 & 3.90 & 3.94 & 0.052 & 0.065 & 0.078 & 0.151 \\
\hline & & $\bar{x}$ & 4.15 & 4.03 & & & & & \\
\hline & & $\hat{\mathrm{P}}$ & $6.31^{\mathrm{a}}$ & $6.53^{a}$ & $6.42^{\mathrm{a}}$ & & & & \\
\hline \multirow{2}{*}{\multicolumn{2}{|c|}{ bacon and ribs }} & M & $5.75^{\mathrm{a}}$ & $4.75^{b}$ & $5.25^{b}$ & 0.063 & $<0.05$ & $<0.05$ & 0.154 \\
\hline & & $\bar{x}$ & 6.03 & 5.64 & & & & & \\
\hline \multirow{3}{*}{\multicolumn{2}{|c|}{ fillet }} & $\mathrm{P}$ & 8.24 & 7.75 & $8.00^{b}$ & & & & \\
\hline & & M & 8.66 & 9.34 & $9.00^{a}$ & 0.053 & $<0.05$ & 0.490 & 0.153 \\
\hline & & $\bar{x}$ & 8.45 & 8.55 & & & & & \\
\hline \multirow{4}{*}{-} & & $\mathrm{P}$ & 2.61 & 2.77 & 2.69 & & & & \\
\hline & longissimus & M & 2.82 & 3.02 & 2.92 & 0.591 & 0.053 & 0.058 & 0.060 \\
\hline & & $\bar{x}$ & 2.72 & 2.90 & & & & & \\
\hline & & $\mathrm{P}$ & 1.56 & 1.47 & $1.52^{b}$ & & & & \\
\hline \multirow[t]{3}{*}{-} & small meat & M & 1.86 & 2.37 & $2.12^{\mathrm{a}}$ & 0.051 & $<0.05$ & 0.097 & 0.506 \\
\hline & & $\bar{x}$ & 1.71 & 1.92 & & & & & \\
\hline & & $\mathrm{P}$ & 1.14 & 1.16 & $1.15^{\mathrm{b}}$ & & & & \\
\hline \multirow[t]{3}{*}{-} & bones & M & 1.24 & 1.32 & $1.28^{\mathrm{a}}$ & 0.133 & $<0.05$ & 0.192 & 0.022 \\
\hline & & $\bar{x}$ & 1.19 & 1.24 & & & & & \\
\hline & & $\mathrm{P}$ & 2.93 & 2.35 & 2.64 & & & & \\
\hline \multirow[t]{2}{*}{-} & skin + fat & M & 2.69 & 2.58 & 2.64 & 0.320 & 0.682 & 0.160 & 0.105 \\
\hline & & $\bar{x}$ & 2.81 & 2.47 & & & & & \\
\hline \multirow{3}{*}{\multicolumn{2}{|c|}{ ham with shin }} & $\mathrm{P}$ & 9.58 & 9.79 & $9.69^{b}$ & & & & \\
\hline & & M & 10.2 & 10.03 & $10.12^{\mathrm{a}}$ & 0.377 & $<0.05$ & 0.852 & 0.103 \\
\hline & & $\bar{x}$ & 9.89 & 9.91 & & & & & \\
\hline \multirow{4}{*}{-} & & $\mathrm{P}$ & 8.59 & 8.86 & 8.73 & & & & \\
\hline & ham & M & 9.19 & 8.97 & 9.08 & 0.201 & 0.129 & 0.812 & 0.102 \\
\hline & & $\bar{x}$ & 8.89 & 8.92 & & & & & \\
\hline & & $\hat{\mathrm{P}}$ & 6.28 & 6.68 & $6.48^{b}$ & & & & \\
\hline \multirow{3}{*}{\multicolumn{2}{|c|}{ - $\quad$ meat }} & M & 6.78 & 6.23 & $6.51^{\mathrm{a}}$ & 0.372 & $<0.05$ & 0.626 & 0.104 \\
\hline & & $\bar{x}$ & 6.53 & 6.46 & & & & & \\
\hline & & $\mathrm{P}$ & 0.61 & 0.62 & 0.62 & & & & \\
\hline & bones & M & 0.66 & 0.66 & 0.66 & 0.520 & 0.054 & 0.146 & 0.009 \\
\hline & & $\bar{x}$ & 0.64 & 0.64 & & & & & \\
\hline & & $\mathrm{P}$ & 1.7 & 1.56 & 1.63 & & & & \\
\hline & skin + fat & M & 1.62 & 1.56 & 1.59 & 0.877 & 0.581 & 0.383 & 0.052 \\
\hline & & $\bar{x}$ & 1.66 & 1.56 & & & & & \\
\hline & & $\mathrm{P}$ & 0.99 & 0.93 & 0.96 & & & & \\
\hline \multirow[t]{2}{*}{-} & shin & M & 1.02 & 1.06 & 1.04 & 0.106 & 0.066 & 0.152 & 0.011 \\
\hline & & $\bar{x}$ & 1.01 & 1.00 & & & & & \\
\hline
\end{tabular}

J-rations with barley (the only cereal grain), O-rations with naked oats, B-breed, F-diet, SEM-standard error of the mean, $n=10$, $\mathrm{ab}-$ means with different superscripts are significantly different at $p \leq 0.05$.

Compared to Pulawska pigs, crossbreds featured a higher $(p \leq 0.05)$ weight of fillet (by $12 \%$ ) and ham with shin (by $4.5 \%$ ) but a significantly lower weight of bacon with ribs (by $18 \%$ ). In addition, an interaction effect of both experimental factors was observed for the weight of bacon with ribs-the weight of this meat cutting was lower $(p \leq 0.05)$ in crossbred pigs receiving rations with naked oats than in other groups.

The pigs' diet showed no impact on the evaluated muscles, except reduced (by 2.35 points) colour lightness (L) of the musculus semimembranosus in pigs receiving feed rations with a $60 \%$ share of naked oats in comparison to those receiving feed rations containing barley (Table 5). 
Table 5. Physical properties of meat.

\begin{tabular}{|c|c|c|c|c|c|c|c|c|}
\hline \multirow{2}{*}{ Item } & \multirow{2}{*}{ Breed (B) } & \multicolumn{2}{|c|}{ Diet (F) } & \multirow[t]{2}{*}{$\overline{\mathbf{x}}$} & \multicolumn{3}{|c|}{$p$-Value } & \multirow{2}{*}{ SEM } \\
\hline & & $\mathbf{J}$ & O & & F & B & F/B & \\
\hline \multicolumn{9}{|c|}{ Longissimus lumborum } \\
\hline \multirow{3}{*}{$\mathrm{pH}_{45}$} & $\mathrm{P}$ & 6.23 & 6.36 & 6.30 & & & & \\
\hline & M & 6.15 & 6.28 & 6.22 & 0.069 & 0.347 & 0.902 & 0.039 \\
\hline & $\bar{x}$ & 6.19 & 6.32 & & & & & \\
\hline \multirow{3}{*}{$\mathrm{pH}_{24}$} & $\mathrm{P}$ & 5.62 & 5.58 & 5.60 & & & & \\
\hline & M & 5.65 & 5.54 & 5.60 & 0.101 & 0.288 & 0.879 & 0.035 \\
\hline & $\bar{x}$ & 5.64 & 5.56 & & & & & \\
\hline \multirow{3}{*}{ WHC (\%) } & $\mathrm{P}$ & 19.6 & 21.3 & $20.4^{b}$ & & & & \\
\hline & M & 22.5 & 24.7 & $23.6^{\mathrm{a}}$ & 0.148 & $<0.05$ & 0.813 & 0.699 \\
\hline & $\bar{x}$ & 21.1 & 23.0 & & & & & \\
\hline \multicolumn{9}{|l|}{ Colour } \\
\hline \multirow{4}{*}{$L^{*}$} & $\mathrm{P}$ & 51.3 & 51.2 & 51.2 & & & & \\
\hline & M & 52.2 & 52.6 & 52.4 & 0.986 & 0.929 & 0.279 & 0.457 \\
\hline & $\bar{x}$ & 51.7 & 51.9 & & & & & \\
\hline & $\mathrm{P}$ & 9.18 & 8.16 & $8.67^{\mathrm{a}}$ & & & & \\
\hline \multirow[t]{3}{*}{$a^{*}$} & M & 7.63 & 7.62 & $7.63^{b}$ & 0.109 & $<0.05$ & 0.114 & 0.180 \\
\hline & $\bar{x}$ & 8.41 & 7.89 & & & & & \\
\hline & $\mathrm{P}$ & 0.768 & 0.757 & 0.763 & & & & \\
\hline \multirow[t]{3}{*}{$b^{*}$} & M & 1.51 & 0.818 & 1.164 & 0.105 & 0.066 & 0.119 & 0.115 \\
\hline & $\bar{x}$ & 1.139 & 0.788 & & & & & \\
\hline & $\mathrm{P}$ & 9.23 & 8.21 & $8.72^{\mathrm{a}}$ & & & & \\
\hline \multirow[t]{3}{*}{$\mathrm{C}=\left[\left(\mathrm{a}^{*}\right)^{2}+\left(\mathrm{b}^{*}\right)^{2}\right]^{0.5}$} & M & 7.82 & 7.68 & $7.75^{b}$ & 0.072 & $<0.05$ & 0.172 & 0.179 \\
\hline & $\bar{x}$ & 8.53 & 7.95 & & & & & \\
\hline & $\mathrm{P}$ & $0.081^{b}$ & $0.092^{b}$ & $0.087^{b}$ & & & & \\
\hline \multirow[t]{2}{*}{$\mathrm{H}=\log \left(\mathrm{b}^{*} / \mathrm{a}^{*}\right)$} & M & $0.200^{\mathrm{a}}$ & $0.101^{b}$ & $0.151^{\mathrm{a}}$ & 0.093 & $<0.05$ & $<0.05$ & 0.015 \\
\hline & $\bar{x}$ & 0.141 & 0.097 & & & & & \\
\hline \multicolumn{9}{|c|}{ Semimembranosus } \\
\hline \multirow{3}{*}{ WHC (\%) } & $\mathrm{P}$ & 19.7 & 17.5 & $18.6^{\mathrm{b}}$ & & & & \\
\hline & M & 21.1 & 20.8 & $20.9^{a}$ & 0.225 & $<0.05$ & 0.102 & 0.429 \\
\hline & $\bar{x}$ & 20.4 & 19.1 & & & & & \\
\hline \multicolumn{9}{|l|}{ Colour } \\
\hline \multirow{4}{*}{$L^{*}$} & $\mathrm{P}$ & 45.9 & 44.6 & $45.2^{b}$ & & & & \\
\hline & M & 49.5 & 46.1 & $47.8^{\mathrm{a}}$ & $<0.05$ & $<0.05$ & 0.288 & 0.556 \\
\hline & $\bar{x}$ & $47.7^{\mathrm{a}}$ & $45.4^{\mathrm{b}}$ & & & & & \\
\hline & $\mathrm{P}$ & 11.4 & 11.9 & 11.7 & & & & \\
\hline \multirow[t]{3}{*}{$a^{*}$} & M & 10.8 & 11.6 & 11.2 & 0.107 & 0.215 & 0.724 & 0.199 \\
\hline & $\bar{x}$ & 11.1 & 11.8 & & & & & \\
\hline & $\mathrm{P}$ & 1.34 & 0.919 & 1.130 & & & & \\
\hline$b^{*}$ & M & 0.838 & 0.879 & 0.858 & 0.562 & 0.415 & 0.500 & 0.163 \\
\hline & $\bar{x}$ & 1.089 & 0.899 & & & & & \\
\hline & $\mathrm{P}$ & 11.6 & 12.0 & 11.8 & & & & \\
\hline$C=\left[\left(a^{*}\right)^{2}+\left(b^{*}\right)^{2}\right]^{0.5}$ & M & 10.9 & 11.6 & 11.3 & 0.153 & 0.174 & 0.676 & 0.194 \\
\hline & $\bar{x}$ & 11.3 & 11.8 & & & & & \\
\hline & $\mathrm{P}$ & 0.121 & 0.071 & 0.096 & & & & \\
\hline $\mathrm{H}=\log \left(\mathrm{b}^{*} / \mathrm{a}^{*}\right)$ & M & 0.091 & 0.071 & 0.081 & 0.358 & 0.592 & 0.601 & 0.015 \\
\hline & $\bar{x}$ & 0.106 & 0.071 & & & & & \\
\hline
\end{tabular}

J-rations with barley (the only cereal grain), O-rations with naked oats, B-breed, F-diet, SEM-standard error of the mean, $n=10$, ab-means with different superscripts are significantly different at $p \leq 0.05$, $\mathrm{L}^{*}$ - lightness, $\mathrm{a}^{*}$-redness, $\mathrm{b}$ $\mathrm{H}$-hue, WHC-water-holding capacity.

The musculus longissimus lumborum and musculus semimembranosus in Pulawska pigs in comparison to crossbred pigs featured significantly improved $(p \leq 0.05)$ water-holding capacity (by 13\% and 11\%) and reduced colour lightness (by 1.2 and 2.6 points). In addition, the longissimus lumborum muscle of Pulawska pigs had a higher (by 0.97) chroma (C) and a lower (by 0.063 ) hue $(\mathrm{H})$ in comparison to Pulawska x PLW pigs. Analysing the impact 
of breed and diet, an interaction $(p \leq 0.05)$ was observed for the hue of the longissimus lumborum muscle.

The content of essential nutrients in musculus longissimus lumborum and musclus semimembranosus did not depend on the pigs' diet and breed, or on their interaction (F/B) except that a higher (by $18 \% ; p \leq 0.05$ ) content of intramuscular fat was measured in the longissimus lumborum muscles of Pulawska pigs in comparison to those of crossbred pigs (Table 6).

Table 6. Chemical composition (g/100 g) of meat.

\begin{tabular}{|c|c|c|c|c|c|c|c|c|}
\hline \multirow{2}{*}{ Item } & \multirow{2}{*}{ Breed (B) } & \multicolumn{2}{|c|}{ Diet (F) } & \multirow[t]{2}{*}{$\overline{\mathbf{x}}$} & \multicolumn{3}{|c|}{$p$-Value } & \multirow{2}{*}{ SEM } \\
\hline & & $\mathbf{J}$ & $\mathbf{O}$ & & $\mathbf{F}$ & B & F/B & \\
\hline \multicolumn{9}{|c|}{ Longissimus lumborum } \\
\hline \multirow{3}{*}{ Dry matter } & $\mathrm{P}$ & 25.54 & 25.98 & 25.76 & & & & \\
\hline & M & 25.89 & 25.63 & 25.76 & 0.266 & 0.105 & 0.105 & 0.140 \\
\hline & $\bar{x}$ & 25.71 & 25.80 & & & & & \\
\hline \multirow{3}{*}{ Crude ash } & $\mathrm{P}$ & 1.13 & 1.11 & 1.12 & & & & \\
\hline & M & 1.11 & 1.16 & 1.13 & 0.129 & 0.194 & 0.059 & 0.005 \\
\hline & $\bar{x}$ & 1.12 & 1.13 & & & & & \\
\hline \multirow{3}{*}{ Crude protein } & $\mathrm{P}$ & 22.65 & 22.79 & 22.72 & & & & \\
\hline & M & 22.66 & 22.25 & 22.46 & 0.391 & 0.106 & 0.095 & 0.840 \\
\hline & $\bar{x}$ & 22.66 & 22.52 & & & & & \\
\hline \multirow{3}{*}{ Crude fat } & $\mathrm{P}$ & 1.75 & 2.08 & $1.91^{\mathrm{a}}$ & & & & \\
\hline & M & 2.01 & 1.22 & $1.61^{b}$ & 0.716 & $<0.05$ & 0.183 & 0.114 \\
\hline & $\bar{x}$ & 1.88 & 1.65 & & & & & \\
\hline \multicolumn{9}{|c|}{ Semimembranosus } \\
\hline \multirow{4}{*}{ Dry matter } & $\mathrm{P}$ & 24.69 & 24.86 & 24.78 & & & & \\
\hline & M & 25.00 & 24.60 & 24.80 & 0.800 & 0.256 & 0439 & 0.134 \\
\hline & $\bar{x}$ & 24.85 & 24.73 & & & & & \\
\hline & $\mathrm{P}$ & 1.10 & 1.09 & 1.10 & & & & \\
\hline \multirow[t]{2}{*}{ Crude ash } & M & 1.09 & 1.11 & 1.10 & 0.845 & 0.559 & 0131 & 0.005 \\
\hline & $\bar{x}$ & 1.10 & 1.10 & & & & & \\
\hline \multirow{3}{*}{ Crude protein } & $\mathrm{P}$ & 22.04 & 21.97 & 22.01 & & & & \\
\hline & M & 21.95 & 21.92 & 21.94 & 0.770 & 0.660 & 0.906 & 0.073 \\
\hline & $\bar{x}$ & 22.00 & 21.95 & & & & & \\
\hline \multirow{3}{*}{ Crude fat } & $\mathrm{P}$ & 1.53 & 1.76 & 1.65 & & & & \\
\hline & M & 1.93 & 1.25 & 1.59 & 0.885 & 0.161 & 0.170 & 0.091 \\
\hline & $\bar{x}$ & 1.73 & 1.51 & & & & & \\
\hline
\end{tabular}

J-rations with barley (the only cereal grain), O-rations with naked oats, B-breed, F-diet, SEM-standard error of the mean, $n=5$. $\mathrm{ab}-$ means with different superscripts are significantly different at $p \leq 0.05$.

Analysis of the lipid profile of intramuscular fat in pigs' longissimus lumborum and semimembranosus muscles showed a significant impact of both experimental factors and their interactions (Tables 7 and 8). 
Table 7. Fatty acid profile (\% total FA) of longissimus lumborum meat.

\begin{tabular}{|c|c|c|c|c|c|c|c|c|}
\hline \multirow{2}{*}{ Item } & \multirow{2}{*}{ Breed (B) } & \multicolumn{2}{|c|}{ Diet (F) } & \multirow[t]{2}{*}{$\overline{\mathbf{x}}$} & \multicolumn{3}{|c|}{$p$-Value } & \multirow{2}{*}{ SEM } \\
\hline & & $\mathbf{J}$ & O & & $\mathbf{F}$ & B & F/B & \\
\hline \multirow{4}{*}{ C 14:0 } & $\mathrm{P}$ & 1.01 & 1.00 & 1.01 & & & & \\
\hline & M & 1.01 & 0.91 & 0.96 & 0.082 & 0.132 & 0.183 & 0.017 \\
\hline & $\bar{x}$ & 1.01 & 0.96 & & & & & \\
\hline & $\mathrm{P}$ & $27.93^{\mathrm{ab}}$ & $28.50^{\mathrm{a}}$ & 28.22 & & & & \\
\hline \multirow[t]{3}{*}{ C 16:0 } & M & $28.46^{\mathrm{a}}$ & $26.70^{b}$ & 27.58 & 0.206 & 0.179 & $<0.05$ & 0.265 \\
\hline & $\bar{x}$ & 28.20 & 27.60 & & & & & \\
\hline & $\mathrm{P}$ & $10.37^{b}$ & $10.90^{\mathrm{a}}$ & 10.64 & & & & \\
\hline \multirow[t]{3}{*}{ C 18:0 } & M & $10.96^{\mathrm{a}}$ & $10.21^{\mathrm{b}}$ & 10.59 & 0.980 & 0.652 & $<0.05$ & 0.171 \\
\hline & $\bar{x}$ & 10.67 & 10.56 & & & & & \\
\hline & $\mathrm{P}$ & $52.63^{\mathrm{a}}$ & $51.32^{\mathrm{ab}}$ & 51.98 & & & & \\
\hline \multirow[t]{3}{*}{ C 18:1 n-9 } & M & $50.52^{b}$ & $51.98^{a b}$ & 51.25 & 0.948 & 0.157 & $<0.05$ & 0.267 \\
\hline & $\bar{x}$ & 51.58 & 51.65 & & & & & \\
\hline & $\mathrm{P}$ & 2.68 & 3.39 & $3.04^{b}$ & & & & \\
\hline \multirow[t]{3}{*}{ C 18:2n-6 } & M & 4.08 & 5.32 & $4.70^{\mathrm{a}}$ & $<0.05$ & $<0.05$ & 0.497 & 0.283 \\
\hline & $\bar{x}$ & $3.38^{b}$ & $4.36^{\mathrm{a}}$ & & & & & \\
\hline & $\mathrm{P}$ & 0.07 & 0.11 & 0.09 & & & & \\
\hline \multirow{3}{*}{ C $18: 3 n-3$} & M & 0.07 & 0.14 & 0.11 & $<0.05$ & 0.489 & 0.611 & 0.011 \\
\hline & $\bar{x}$ & $0.07^{\mathrm{b}}$ & $0.13^{a}$ & & & & & \\
\hline & $\mathrm{P}$ & 0.07 & 0.06 & 0.07 & & & & \\
\hline \multirow[t]{3}{*}{ C 20:0 } & M & 0.06 & 0.05 & 0.06 & 0.205 & 0.474 & 0.665 & 0.003 \\
\hline & $\overline{\mathrm{x}}$ & 0.07 & 0.06 & & & & & \\
\hline & $\mathrm{P}$ & 0.33 & 0.33 & 0.33 & & & & \\
\hline \multirow[t]{3}{*}{ C 20:1 n-9 } & M & 0.31 & 0.28 & 0.30 & 0.646 & 0.230 & 0.646 & 0.012 \\
\hline & $\bar{x}$ & 0.32 & 0.31 & & & & & \\
\hline & $\mathrm{P}$ & 0.06 & 0.06 & 0.06 & & & & \\
\hline \multirow{3}{*}{ C 20:2 n-6 } & $\mathrm{M}$ & 0.06 & 0.08 & 0.07 & 0.449 & 0.240 & 0.333 & 0.004 \\
\hline & $\bar{x}$ & 0.06 & 0.07 & & & & & \\
\hline & $\mathrm{P}$ & $0.03^{a}$ & $0.02^{b}$ & 0.03 & & & & \\
\hline \multirow[t]{3}{*}{ C $20: 3 n-3$} & M & $0.02^{b}$ & $0.03^{a}$ & 0.03 & 0.600 & 0.600 & $<0.05$ & 0.001 \\
\hline & $\bar{x}$ & 0.03 & 0.03 & & & & & \\
\hline & $\mathrm{P}$ & $0.21^{a b}$ & $0.18^{b}$ & $0.20^{b}$ & & & & \\
\hline \multirow{3}{*}{ C 20:4 n-6 } & M & $0.22^{a b}$ & $0.32^{\mathrm{a}}$ & $0.27^{\mathrm{a}}$ & 0.233 & $<0.05$ & $<0.05$ & 0.018 \\
\hline & $\bar{x}$ & 0.22 & 0.25 & & & & & \\
\hline & $P$ & $39.44^{\mathrm{ab}}$ & $40.70^{a}$ & 40.07 & & & & \\
\hline SFA & M & $40.53^{\mathrm{a}}$ & $37.92^{\mathrm{b}}$ & 39.23 & 0.321 & 0.218 & $<0.05$ & 0.395 \\
\hline & $\bar{x}$ & 39.99 & 39.31 & & & & & \\
\hline & $\mathrm{P}$ & $60.39^{a b}$ & $59.40^{\mathrm{b}}$ & 59.90 & & & & \\
\hline UFA & M & $59.28^{\mathrm{b}}$ & $61.87^{a}$ & 60.58 & 0.301 & 0.250 & $<0.05$ & 0.389 \\
\hline & $\bar{x}$ & 59.84 & 60.64 & & & & & \\
\hline & $\mathrm{P}$ & $57.35^{\mathrm{a}}$ & $55.65^{\mathrm{b}}$ & $56.50^{a}$ & & & & \\
\hline MUFA & M & $54.83^{\mathrm{b}}$ & $55.99^{\mathrm{b}}$ & $55.41^{\mathrm{b}}$ & 0.418 & $<0.05$ & $<0.05$ & 0.296 \\
\hline & $\bar{x}$ & 56.09 & 55.82 & & & & & \\
\hline & $\mathrm{P}$ & 3.04 & 3.75 & $3.40^{b}$ & & & & \\
\hline PUFA & M & $4.45^{b}$ & $5.88^{a}$ & $5.17^{\mathrm{a}}$ & $<0.05$ & $<0.05$ & 0.404 & 0.308 \\
\hline & $\bar{x}$ & 3.75 & 4.82 & & & & & \\
\hline & $\hat{\mathrm{P}}$ & $70.76^{\mathrm{ab}}$ & $70.50^{\mathrm{b}}$ & 70.63 & & & & \\
\hline DFA & M & $70.24^{\mathrm{b}}$ & $72.08^{a}$ & 71.16 & 0.151 & 0.187 & $<0.05$ & 0.270 \\
\hline & $\bar{x}$ & 70.50 & 71.29 & & & & & \\
\hline & $\mathrm{P}$ & $28.94^{\mathrm{ab}}$ & $29.50^{a}$ & 29.22 & & & & \\
\hline OFA & M & $29.46^{a}$ & $27.61^{b}$ & 28.54 & 0.182 & 0.162 & $<0.05$ & 0.276 \\
\hline & $\bar{x}$ & 29.20 & 28.56 & & & & & \\
\hline & $\mathrm{P}$ & 29.5 & 29.3 & $29.40^{b}$ & & & & \\
\hline$n-6 / n-3$ & M & 47.4 & 41.5 & $44.45^{\mathrm{a}}$ & 0.570 & $<0.05$ & 0.596 & 3.00 \\
\hline & $\bar{x}$ & 38.45 & 35.40 & & & & & \\
\hline
\end{tabular}

J-rations with barley (the only cereal grain), O-rations with naked oats, B-breed, F-diet, SEM-standard error of the mean, $n=5$, $\mathrm{ab}$ - means with different superscripts are significantly different at $p \leq 0.05$, SFA—saturated fatty acids, UFA—unsaturated fatty acids, MUFA - monounsaturated fatty acids, PUFA - polyunsaturated fatty acids, DFA — neutral or hypocholesterolemic fatty acids = MUFA +

C18:0, OFA - hypercholesterolemic fatty acids $=$ C14:0 + C16:0 . 
Table 8. Fatty acid profiles (\% total FA) of semimembranosus meat.

\begin{tabular}{|c|c|c|c|c|c|c|c|c|}
\hline \multirow{2}{*}{ Item } & \multirow{2}{*}{ Breed (B) } & \multicolumn{2}{|c|}{ Diet (F) } & \multirow{2}{*}{$\overline{\mathbf{x}}$} & \multicolumn{3}{|c|}{$p$-Value } & \multirow{2}{*}{ SEM } \\
\hline & & $\mathbf{J}$ & O & & $\mathbf{F}$ & B & $\mathrm{F} / \mathrm{B}$ & \\
\hline \multirow{4}{*}{ C 14:0 } & $\mathrm{P}$ & 0.92 & 0.92 & $0.92^{\mathrm{a}}$ & & & & \\
\hline & M & 0.87 & 0.83 & $0.85^{b}$ & 0.570 & $<0.05$ & 0.523 & 0.015 \\
\hline & $\bar{x}$ & 0.90 & 0.88 & & & & & \\
\hline & $\mathrm{P}$ & 25.51 & 25.32 & 25.42 & & & & \\
\hline \multirow[t]{3}{*}{ C 16:0 } & M & 25.45 & 25.47 & 25.46 & 0.681 & 0.829 & 0.634 & 0.098 \\
\hline & $\bar{x}$ & 25.48 & 25.40 & & & & & \\
\hline & $\mathrm{P}$ & 9.7 & 9.27 & 9.49 & & & & \\
\hline \multirow[t]{3}{*}{ C 18:0 } & M & 9.6 & 9.38 & 9.49 & 0.154 & 0.971 & 0.644 & 0.108 \\
\hline & $\bar{x}$ & 9.65 & 9.33 & & & & & \\
\hline & $\mathrm{P}$ & 53.14 & 53.03 & 53.09 & & & & \\
\hline \multirow{3}{*}{ C 18:1 n-9 } & $\mathrm{M}$ & 52.91 & 52.33 & 52.62 & 0.466 & 0.365 & 0.633 & 0.232 \\
\hline & $\bar{x}$ & 53.03 & 52.68 & & & & & \\
\hline & $\mathrm{P}$ & 4.66 & 5.79 & $5.23^{b}$ & & & & \\
\hline \multirow[t]{3}{*}{ C 18:2 n-6 } & M & 5.6 & 6.86 & $6.23^{\mathrm{a}}$ & $<0.05$ & $<0.05$ & 0.847 & 0.240 \\
\hline & $\bar{x}$ & $5.13^{b}$ & $6.33^{a}$ & & & & & \\
\hline & $\mathrm{P}$ & 0.09 & 0.15 & 0.12 & & & & \\
\hline \multirow{3}{*}{ C $18: 3 n-3$} & $\mathrm{M}$ & 0.1 & 0.09 & 0.10 & 0.123 & 0.057 & 0.051 & 0.008 \\
\hline & $\bar{x}$ & 0.10 & 0.12 & & & & & \\
\hline & $\mathrm{P}$ & $0.06^{\mathrm{a}}$ & $0.02^{b}$ & $0.04^{\mathrm{b}}$ & & & & \\
\hline \multirow[t]{3}{*}{ C 20:0 } & M & $0.06^{\mathrm{a}}$ & $0.05^{\mathrm{a}}$ & $0.06^{\mathrm{a}}$ & $<0.05$ & $<0.05$ & $<0.05$ & 0.003 \\
\hline & $\bar{x}$ & $0.06^{\mathrm{a}}$ & $0.04^{\mathrm{b}}$ & & & & & \\
\hline & $\mathrm{P}$ & 0.32 & 0.3 & 0.31 & & & & \\
\hline \multirow{3}{*}{ C 20:1 n-9 } & $\mathrm{M}$ & 0.30 & 0.27 & 0.29 & 0.109 & 0.065 & 0.677 & 0.007 \\
\hline & $\bar{x}$ & 0.31 & 0.29 & & & & & \\
\hline & $\mathrm{P}$ & $0.09^{a}$ & $0.06^{b}$ & $0.08^{b}$ & & & & \\
\hline \multirow[t]{3}{*}{ C 20:2 n-6 } & M & $0.10^{\mathrm{a}}$ & $0.11^{\mathrm{a}}$ & $0.11^{\mathrm{a}}$ & 0.115 & $<0.05$ & $<0.05$ & 0.005 \\
\hline & $\bar{x}$ & 0.10 & 0.09 & & & & & \\
\hline & $\mathrm{P}$ & 0.04 & 0.04 & 0.04 & & & & \\
\hline \multirow[t]{3}{*}{ C 20:3 n-3 } & M & 0.04 & 0.05 & 0.05 & 0.569 & 0.346 & 0.703 & 0.004 \\
\hline & $\bar{x}$ & 0.04 & 0.05 & & & & & \\
\hline & $\mathrm{P}$ & 0.47 & 0.39 & $0.43^{b}$ & & & & \\
\hline \multirow[t]{3}{*}{ C 20:4 n-6 } & M & 0.51 & 0.63 & $0.57^{\mathrm{a}}$ & 0.758 & $<0.05$ & 0.117 & 0.035 \\
\hline & $\overline{\mathrm{x}}$ & 0.49 & 0.51 & & & & & \\
\hline & $\mathrm{P}$ & 36.23 & 35.58 & 35.91 & & & & \\
\hline SFA & M & 36.03 & 35.77 & 35.90 & 0.262 & 0.986 & 0.621 & 0.190 \\
\hline & $\bar{x}$ & 36.13 & 35.68 & & & & & \\
\hline & $\mathrm{P}$ & 63.54 & 64.17 & 63.86 & & & & \\
\hline UFA & M & 63.75 & 64 & 63.88 & 0.277 & 0.956 & 0.624 & 0.188 \\
\hline & $\bar{x}$ & 63.65 & 64.09 & & & & & \\
\hline & $\mathrm{P}$ & 58.17 & 57.75 & 57.96 & & & & \\
\hline MUFA & M & 57.39 & 56.26 & 56.83 & 0.173 & 0.053 & 0.521 & 0.298 \\
\hline & $\bar{x}$ & 57.78 & 57.01 & & & & & \\
\hline & $\mathrm{P}$ & 5.36 & 6.42 & $5.89^{b}$ & & & & \\
\hline PUFA & M & 6.36 & 7.74 & $7.05^{\mathrm{a}}$ & $<0.05$ & $<0.05$ & 0.676 & 0.260 \\
\hline & $\bar{x}$ & $5.86^{\mathrm{b}}$ & $7.08^{a}$ & & & & & \\
\hline & $\mathrm{P}$ & 73.24 & 73.44 & 73.34 & & & & \\
\hline DFA & M & 73.36 & 73.38 & 73.37 & 0.622 & 0.894 & 0.685 & 0.103 \\
\hline & $\bar{x}$ & 73.30 & 73.41 & & & & & \\
\hline & $\mathrm{P}$ & 26.43 & 26.24 & 26.34 & & & & \\
\hline OFA & M & 26.32 & 26.3 & 26.31 & 0.649 & 0.923 & 0.721 & 0.104 \\
\hline & $\bar{x}$ & 26.38 & 26.27 & & & & & \\
\hline & $\mathrm{P}$ & $37.1^{\mathrm{b}}$ & $35.0^{\mathrm{b}}$ & $36.05^{b}$ & & & & \\
\hline$n-6 / n-3$ & M & $45.6^{\mathrm{b}}$ & $58.2^{\mathrm{a}}$ & $51.90^{\mathrm{a}}$ & 0.062 & $<0.05$ & $<0.05$ & 2.29 \\
\hline & $\overline{\mathrm{x}}$ & 38.65 & 44.10 & & & & & \\
\hline
\end{tabular}

J-rations with barley (the only cereal grain), O-rations with naked oats, B-breed, F-diet, SEM-standard error of the mean, $n=5$, $\mathrm{ab}$ - means with different superscripts are significantly different at $p \leq 0.05$, SFA—saturated fatty acids, UFA—unsaturated fatty acids, MUFA - monounsaturated fatty acids, PUFA - polyunsaturated fatty acids, DFA—neutral or hypocholesterolemic fatty acids = MUFA + C18:0, OFA—hypercholesterolemic fatty acids $=$ C14:0 + C16:0.

Naked oats included in the pigs' diet increased (by $28 \%$ in $m$. longissimus lumborum and by $20 \%$ in $m$. semimembranosus) the share of PUFA $(p \leq 0.05)$. In addition, the muscles of Pulawska x PLW pigs contained more by 51 and $19 \%(p \leq 0.05)$ polyunsaturated fatty acids (PUFA) than the muscles of the Pulawska breed in the longissimus lumborum and in 
the semimembranosus, respectively. The muscles of Pulawska pigs showed a significantly improved n-6/n-3 acid ratio by 66 and $69 \%$ in $m$. longissimus lumborum and m. semimembranosus. In the n- $6 / \mathrm{n}-3$ acid ratio in the semimembranosus muscle, an interaction effect was observed. The pigs' diet and their breed had no significant impact on the total saturated fatty acids (SFA) and total unsaturated fatty acids (UFA) nor on neutral or hypocholesterolemic (DFA) and hypercholesterolemic fatty acids (OFA) in either of the evaluated muscles.

\section{Discussion}

A $60 \%$ inclusion of naked oats in diets for pigs had no impact $(p>0.05)$ on the daily weight gain of pigs. Meanwhile, Brand and Merwe [27], having added naked oats in amounts corresponding to $0,24.25,49.25$, and $73.50 \%$ of pigs' rations $(25.7-89.2 \mathrm{~kg})$, noted a reduction in daily weight gain by 22,30 , and $39 \mathrm{~g}$, respectively, in comparison to control pigs (918 g). At the same time, grower-finisher pigs (Large White $x$ Landrace) receiving naked oats in their feed rations showed an improved feed conversion rate. However, Morris and Burrows [22], evaluating feed rations containing 30,65, and $96.75 \%$ of naked oats, observed an insignificant increase (by 10-20 g) in the daily weight gain of experimental pigs $(($ Hampshire $\times$ Duroc $) \times($ Yorkshire $\times$ Landrace $)$ in comparison to the control ones $(820 \mathrm{~g})$. In turn, Kosieradzka and Fabijańska [48], having replaced $30 \%$ of wheat with naked oats in diets for fattening pigs, recorded a higher $(872.25 \mathrm{~g})$ daily weight gain than in the control group $(866.25 \mathrm{~g})$. Pigs receiving naked oats in feed rations showed a better feed conversion rate $(2.82 \mathrm{~kg})$ than the control pigs $(2.96 \mathrm{~kg})$. Similarly, Fabijańska and Bekta [35] demonstrated improved daily weight gain $(855 \mathrm{~g})$ of pigs ((Polish Large White $\times$ Polish Landrace $) \times($ Duroc $\times$ Pietrain $)$ fed rations in which all barley was replaced by naked oats, with a combined feed conversion rate $(2.81 \mathrm{~kg})$ lower than in the pigs fed rations containing barley. Semeniuk and Grela [49] also observed a positive impact of including $50 \%$ of naked oats in cereal feed materials for fattening pigs ((Polish Landrace $\times$ Polish Large White) $\times$ Pietrain). Variability in the pigs' weight gain and feed conversion rates can be explained by the inclusion of naked oats in their diet and by the fact that the trials involved various commercial crossbred lines.

Lower daily weight gain and a higher feed conversion rate were recorded in Pulawska pigs compared to Pulawska and PLW crossbreds in studies by Stasiak et al. [50] and POLSUS [51]. The daily weight gain of Pulawska pigs and Pulawska x PLW crossbreds throughout the fattening period should be considered good, as Stasiak et al. [50] reported a daily weight gain lower by $635 \mathrm{~g}$ for Pulawska and PLW crossbreds. Similarly, POLSUS [51], when evaluating the usability of pigs, mentioned a lower standard weight gain for sows and hogs of respective breeds: Pulawska: 565 and $575 \mathrm{~g}$ and Polish Large White (PLW): 659 and $763 \mathrm{~g}$.

The present study did not show any significant impact on the pigs' diet for their carcass composition and muscle and fat content, which corroborated the results obtained by Milczarek and Osek [25] involving crossbreds (PLW x PL) x (Duroc x Pietrain). The only exception was that the "eye" of the loin in pigs fed rations containing $60 \%$ of naked oats was about $2 \mathrm{~cm}^{2}$ smaller $(p \leq 0.05)$. Kosieradzka and Fabijańska [48], having replaced $30 \%$ of wheat with naked oats in the feed ration of pigs ((Polish Large White $\times$ Polish Landrace) $\times($ Duroc $\times$ Pietrain) $)$, noted that dressing percentage improved by $0.73 \%$ and the "eye" of the loin increased by $2.22 \mathrm{~cm}^{2}$. At the same time, experimental pigs featured significantly thinner backfat $(25.81 \mathrm{~mm})$ than those from the control group $(29.07 \mathrm{~mm})$. Moreover, Fabijańska et al. [21], having replaced 55\% of barley with naked oats, observed that the "eye" of the loin was bigger $\left(48.64 \mathrm{~cm}^{2}\right)$ than in control pigs $\left(46.86 \mathrm{~cm}^{2}\right)$. However, the backfat of fattening pigs receiving feed rations with oats was $3.11 \mathrm{~mm}$ thicker when measured at five points. In turn, Brand and Merwe [27] did not find any significant differences in dressing percentage, although it increased $(76.2-77.1 \%)$ as the share of naked oats was increased in the feed ration of pigs (Large White $\times$ Landrace). The authors did not record the impact of oats on the content of carcass fat. Similarly, Morris and Burrows [22] 
demonstrated that a higher (65 and 96.75\%) share of naked oats in the pigs' diet significantly (by about $1 \%$ ) improved the dressing percentage when comparing control $(77.4 \%)$ and experimental pigs $(76.9 \%)$ fed rations containing $30 \%$ of naked oats. In our own studies, only the dressing percentage of pigs fed diets containing $60 \%$ of naked oats tended to increase.

Analysing carcass composition, breed was not observed to have an impact on the pigs' dressing percentage, musculature, or fatness - only the "eye" of the loin was significantly smaller in Pulawska pigs compared to Pulawska x PLW crossbreds. In turn, Stasiak et al. [50] recorded a lower $(48.1 \%)$ content of meat in the carcass and thinner $(20.0 \mathrm{~mm})$ backfat in Pulawska x PLW crossbreds. Improved dressing percentage $(79.24 \%)$ and meatiness $(55.47 \%)$, and thinner $(16.9 \mathrm{~mm})$ backfat at five points of measurement, were found by Kasprzyk et al. [11] in Pulawska pigs slaughtered when they reached a weight of about $100 \mathrm{~kg}$. Similarly, Piórkowska et al. [12] noted thinner (19.4 mm) backfat but a bigger "eye" of the loin $\left(48.3 \mathrm{~cm}^{2}\right)$ for a lower slaughter weight. In turn, Babicz et al. [14], slaughtering Pulawska pigs weighing 135-140 kg, found a bigger "eye" of the loin (more than $58 \mathrm{~cm}^{2}$ ) and thicker backfat (nearly $41 \mathrm{~mm}$ ).

The inclusion of $60 \%$ of naked oats in the diets of Pulawska pigs and Pulawska x PLW crossbreds had no impact on the weights of primal cuts of right-side half-carcasses, which was consistent with the results of studies carried out by Milczarek and Osek [25] involving $(\mathrm{PLW} \times \mathrm{PL}) \times($ Duroc $\times$ Pietrain $)$ crossbreds.

The fact that the pigs' diet had no impact on the physical traits of the evaluated muscles, except reduced lightness (L) of the ham muscle of pigs receiving diets with $60 \%$ of naked oats, corresponded to results obtained by Milczarek and Osek [25]. Fabijańska et al. [21] and Milczarek and Osek [25] did not note any impact of naked oats used in feed rations for commercial pigs on the water-holding capacity (WHC) of their meat.

In their studies, the musculus longissimus lumborum and musculus semimembranosus in Pulawska pigs showed significantly improved $(p \leq 0.05)$ water-holding capacity and reduced colour lightness (L) in comparison to crossbreds. According to Kasprzyk et al. [2], native breeds of pigs, including Pulawska pigs, had lower values of $\mathrm{L}$ in their longissimus muscle compared to commercial breeds. Moreover, the longissimus lumborum muscle of Pulawska pigs had a higher chroma (C) and a lower hue (H). Similar (6.25) $\mathrm{pH}_{1}$ and lower (5.59) $\mathrm{pH}_{24}$ values in the longissimus muscle of Pulawska pigs were reported by Piórkowska et al. [12] and Kasprzyk et al. [2]. Sieczkowska et al. [52] and Kasprzyk et al. [11] claimed that the acidification of the muscle tissue at a post mortem time of $24 \mathrm{~h}$ is a very important and significant parameter for meat processing plants. The reported reduction in $\mathrm{pH}$ at a post mortem time of $24 \mathrm{~h}$ is an indicator of normal meat maturation. The reported $\mathrm{pH}_{24}$ values for all the genetic groups were typical of normal meat [53].

The content of essential nutrients in musculus longissimus lumborum and musclus semimembranosus did not depend on the diet and breed of pigs, except that a higher $(p \leq 0.05)$ content of intramuscular fat was found in the longissimus lumborum muscle of Pulawska pigs. The lack of impact of a $60 \%$ inclusion of naked oats in feed rations for pigs on the content of essential nutrients in the longissimus muscle was concurrent with the results of Fabijańska and Bekta [35] and Milczarek and Osek [25]; however, they carried out their studies on commercial (fast-growing) pigs. Florowski et al. [54], comparing the essential nutrient compositions of the longissimus muscle in Pulawska pigs and Polish Landrace (PL) pigs noted a significantly higher dry matter, protein, and intramuscular fat content. In turn, Kasprzyk and Bougnacka [4] showed similar contents (2.78\% vs. 2.50 and $2.51 \%$ ) of fat in the longissimus lumborum muscle of Pulawska pigs compared to DanBred and Naima breeds. Intramuscular fat content is an important indicator of meat quality and its qualification for culinary purposes. According to Wajda et al. [55], Łyczyński et al. [56], and Grześkowiak et al. [57], the optimum level of this component in the longissimus muscle should be 2-3\%. Intramuscular fat (IMF) content in the muscle measured in our own study was relatively low, but similar to that noted in the muscle of Pulawska pigs by Kasprzyk et al. [11] and Piórkowska et al. [12]. A lower $(\leq 1 \%)$ content of intramuscular fat could 
deteriorate the flavour of the meat which, particularly after heat treatment, becomes dry and stringy [58]; on the other hand, a higher content of IMF (above 3.5\%) can contribute to lower evaluations of meat by consumers due to the visible fat deposits [59].

The composition and share of fatty acids in the lipid fraction of muscles testifies to the dietary value of meat $[60,61]$. A positive impact of including naked oats in the feed ration was observed on the fatty acid profile of IMF. A significant increase in the share of PUFA, and particular linoleic acid (C18:2), classified as a so-called essential fatty acid (EFA), was observed in both muscles. These data corroborate the results of previous studies by Milczarek and Osek [25], which also showed increased levels of PUFA in the meat of commercial fattening pigs $((\mathrm{PLW} \times \mathrm{PL}) \times($ Duroc $\times$ Pietrain $))$ fed rations containing naked oats.

The pigs' diet and their breed showed no significant impact on the total saturated fatty acids (SFA) and total unsaturated fatty acids (UFA), nor on neutral or hypocholesterolemic (DFA) and hypercholesterolemic fatty acids (OFA) in either of the evaluated muscles. The muscles of Pulawska $x$ PLW pigs were found to contain more $(p \leq 0.05)$ polyunsaturated fatty acids (PUFA), but the muscles of Pulawska pigs featured a significantly healthier $\mathrm{n}-6 / \mathrm{n}-3$ ratio. Less SFA, but more PUFA, and simultaneously a lower share of PUFA n-3 in the muscles of Pulawska pigs, were measured by Cebulska et al. [62] and Milczarek et al. [3], which can testify to the positive impact of the pigs' diet, and in particular the inclusion of naked oats. Migdał et al. [63] claim that slow-growing pigs show a lipid fraction with an improved fatty acid profile. However, a higher concentration of polyunsaturated fatty acids in intramuscular fat or fat reserves has an adverse effect on the technological and sensory traits of the resulting meat product.

\section{Conclusions}

To sum up, a $60 \%$ inclusion of naked oats in the diets of Pulawska pigs and Pulawska $x$ PLW crossbreds resulted in a similar daily weight gain at a slightly higher feed conversion rate compared to pigs fed rations containing barley as the only grain. The diet had no impact on the carcass composition (dressing percentage, fatness, and musculature), essential nutrient contents, or physical traits of the muscles; however, naked oats in pigs ${ }^{\prime}$ diets significantly increased the share of PUFA in both muscles. Pulawska pigs showed worse fattening and at-slaughter results but their meat was of good quality, with improved water-holding capacity and nutritive value measured as the n-6/n-3 acid ratio.

Author Contributions: Conceptualization, A.M.; methodology, A.M.; formal analysis, A.M.; investigation, A.M.; resources, A.M.; writing—original draft preparation, A.M.; writing—review and editing, A.M.; visualization, A.M.; project administration, A.M.; funding acquisition, A.M. All authors have read and agreed to the published version of the manuscript.

Funding: This research was funded by the Ministry of Science and Higher Education of Poland. Statutory activity: 212/04/S.

Institutional Review Board Statement: Ethical review and approval was waived for this study, as according to Polish law, ethical approval is not required for services within the scope of the Act of 18 December 2003 on animal treatment facilities, as well as agricultural activities, including the rearing or breeding of animals. The research was carried out in accordance with the provisions on the protection of animals, and activities that, in compliance with the practices of veterinary medicine, do not cause pain, suffering, distress, or permanent damage to the body of animals, to an extent equal to or more intense than a needle prick (Act of the Protection of Animals Used for Scientific and Educational Purposes, Legislative Decree 266/2015).

Informed Consent Statement: Not applicable.

Data Availability Statement: Data is available upon request from the corresponding author.

Conflicts of Interest: The author declares no conflict of interest. 


\section{References}

1. OECD/FAO. OECD-FAO Agricultural Outlook; Database; OECD Agriculture Statistics. 2021. Available online: https://stats. oecd.org/viewhtml.aspx?datasetcode=HIGH_AGLINK_2017\&lang=en (accessed on 27 September 2021).

2. Kasprzyk, A.; Tyra, M.; Babicz, M. Fatty acid profile of pork from a local and a commercial breed. Arch. Anim. Breed. 2015, 58, 379-385. [CrossRef]

3. Milczarek, A.; Osek, M.; Banaszkiewicz, T. Chemical composition of meat from the Pulawska breed pigs, depending on their slaughter weight. J. Elem. 2019, 24, 639-648. [CrossRef]

4. Kasprzyk, A.; Bogucka, J. Meat quality of Pulawska breed pigs and image of longissimus lumborum muscle microstructure compared to commercial DanBred and Naima hybrids. Arch. Anim. Breed. 2020, 63, 293-301. [CrossRef]

5. Dehghan, M.; Mente, A.; Zhang, X.; Swaminathan, S.; Li, W.; Mohan, V.; Iqbal, R.; Kumar, R.; Wentzel-Viljoen, E.; Rosengren, A.; et al. Associations of fats and carbohydrate intake with cardiovascular disease and mortality in 18 countries from five continents (PURE): A prospective cohort study. Lancet 2017, 390, 2050-2062. [CrossRef]

6. Nettleton, J.A.; Brouwer, I.A.; Geleijnse, J.M.; Hornstra, G. Saturated fat consumption and risk of coronary heart disease and ischemic stroke: A science update. Ann. Nutr. Metab. 2017, 70, 26-33. [CrossRef]

7. Jaworska, D.; Przybylski, W.; Kołożyn-Krajewska, D.; Czarniecka-Skubina, E.; Wachowicz, I.; Trząskowska, M.; Kajak, K.; Lech, A.; Niemyski, S. The assessment of relationships between characteristics determining technological and sensory quality of pork. Anim. Sci. Pap. Rep. 2006, 24, 121-135.

8. Łyczyński, A.; Pospiech, E.; Bartkowiak, Z.; Urbaniak, M. Meat content and meat quality depending on the genotype and feeding system of pigs. Żywność 2003, 4, 287-298.

9. Milczarek, A.; Osek, M. The rearing, growing and fattening performance, carcass and meat quality of pigs fed naked oats supplemented with enzymes. J. Anim. Feed Sci. 2006, 15 (Suppl. 1), 69-72. [CrossRef]

10. Prasow, M.; Babicz, M.; Domaradzki, P.; Skałecki, P.; Litwińczuk, A.; Kaliniak, A. Slaughter value and meat quality of local pig breeds in Poland. J. Anim. Sci. Biol. Bioecon. 2018, 36, 5-17. [CrossRef]

11. Kasprzyk, A.; Babicz, M.; Kamyk-Kamieński, P.; Lechowski, J. Slaughter value and meat quality of Pulawska and Polish Landrace breeds fatteners. Annales Universitatis Mariae Curie-Skłodowska. Sectio EE Zootechnica 2013, 31, 1-9.

12. Piórkowska, K.; Tyra, M.; Rogoz, M.; Ropka-Molik, K.; Oczkowicz, M.; Różycki, M. Association of the melanocortin-4 receptor (MC4R) with feed intake, growth, fatness and carcass composition in pigs raised in Poland. Meat Sci. 2010, 85, 297-301. [CrossRef] [PubMed]

13. Domaradzki, P.; Skałecki, P.; Prasow, M.; Babicz, M.; Florek, M. Physicochemical properties and lipid oxidation parameters of selected muscles of Puławska breed fatteners during 14-day ageing in vacuum packaging. Med. Weter. 2020, 76, 400-405. [CrossRef]

14. Babicz, M.; Kamyk, P.; Stasiak, A.; Pastwa, M. Opportunities to use Puławska pigs for heavy fattener production. Ann. Anim. Sci. 2009, 9, 259-268.

15. Wojtysiak, D.; Połtowicz, K. Carcass quality, physico-chemical parameters, muscle fibre traits and myosin heavy chain composition of m. longissimus lumborum from Puławska and Polish Large White pigs. Meat Sci. 2014, 97, 395-403. [CrossRef] [PubMed]

16. Bocian, M.; Wojtysiak, D.; Jankowiak, H.; Cebulska, A.; Kapelański, W.; Migdał, W. Carcass, meat quality and histochemical traits of m. longissimus lumborum from Zlotnicka Spotted pigs and commercial pigs. Folia Biol. Kraków 2012, 60, 181-187. [CrossRef]

17. Debrecéni, O.; Komová, P.; Bučko, O. Comparison the physicochemical quality indicators of Musculus Longissimus Dorsi from Mangalitsa breed and their crossbreeds. J. Cent. Eur. Agric. 2016, 17, 1253-1263. [CrossRef]

18. Matoušek, V.; Kernerová, N.; Hyšplerová, K.; Jirotková, D.; Brzáková, M. Carcass traits and meat quality of Prestice Black-Pied pig breed. Asian Australas. J. Anim. Sci. 2016, 29, 1181-1187.

19. Colonna, M.A.; Tarricone, S.; Giannico, F.; Selvaggi, M.; Carriero, F.; Crupi, P.; Corbo, F.; Clodoveo, M.L. Dietary Effects of Extra Virgin Olive Oil Extracted by Ultrasound Technology or Refined Olive Oil onthe Quality Traits of Pork and “Capocollo di Martina Franca" Dry-Cured Meat. Animals 2021, 11, 954. [CrossRef]

20. Milczarek, A.; Osek, M. Partial replacement of soya bean with low-tannin faba bean varieties (Albus or Amulet): Effects on growth traits, slaughtering parameters and meat quality of Pulawska pigs. Ann. Anim Sci. 2016, 16, 477-487. [CrossRef]

21. Fabijańska, M.; Bekta, M.; Sokół, J.L.; Bobel, B. Selected parameters of slaughter analysis in fatteners fed mixtures containing naked oats with or without the addition of synthetic zeolite. J. Anim. Feed Sci. 2001, 10, 209-214. [CrossRef]

22. Morris, J.R.; Burrows, V.D. Naked oats in grower-Finisher pig diets. Can. J. Anim. Sci. 1986, 66, 833-836. [CrossRef]

23. Thacker, P.A.; Kirkwood, R.N. Nontraditional Feed Sources for Use in Swine Production; CRC Press Taylor \& Francis Group: Boca Raton, FL, USA; London, UK; New York, NY, USA, 2018.

24. Turyk, Z.; Osek, M.; Milczarek, A.; Janocha, A. Meat chemical composition and blood serum lipids of pigs fed mixtures containing barley or triticale. Sci. Ann. Pol. Soc. Anim. Prod. 2015, 11, 71-79.

25. Milczarek, A.; Osek, M. Influence of high naked oats share in mixture on fattening results, slaughter value and meat quality of pigs. Acta Sci. Pol. Zootech. 2009, 8, 27-38.

26. Biel, W.; Jacyno, E.; Kawęcka, M. Chemical composition of hulled, dehulled and naked oat grains. S. Afr. J. Anim. Sci. 2014, 44, 189-197. [CrossRef]

27. Brand, T.S.; Merwe, J.P. Naked oats (Avena nuda) as a substitute for maize in diets for weanling and grower-finisher pigs. Anim. Feed Sci. Technol. 1996, 57, 139-147. [CrossRef] 
28. Krasilnikov, V.N.; Batalova, G.A.; Popov, V.S.; Sergeyevaa, S.S. Fatty Acid Composition of Lipids in Naked Oat Grain of Domestic Varieties. Russ. Agric. Sci. 2018, 44, 406-408. [CrossRef]

29. Koŭrimská, L.; Pokhrel, K.; Božik, B.; Tilami, S.K.; Horčička, P. Fat content and fatty acid profiles of recently registered varieties of naked and hulled oats with and without husks. J. Cereal Sci. 2021, 99, 103216. [CrossRef]

30. Kosieradzka, I.; Fabijańska, M. Wpływ betaglukanów owsa nagiego na jego wartość energetyczną dla kurcząt brojlerów. In Proceedings of the XXIXth Session of Animal Nutrition, Committee on Animal Sciences, Polish Academy of Sciences. "Żywienie Jako Czynnik Modyfikujący Przemiany w Łańcuchu Troficznym: Pasza—Zwierzę-Człowiek”, Rogów, Poland, 27-28 September 2000; p. 32.

31. Redaelli, R.; Sgrulletta, D.; Scalfati, G.; De Stefanis, E.; Cacciatori, P. Naked Oats for Improving Human Nutrition: Genetic and Agronomic Variability of Grain Bioactive Components. Crop Sci. 2009, 49, 1431-1437. [CrossRef]

32. Demibras, A. B-Glucan and mineral nutrient contents of cereals grown in Turkey. Food Chem. 2005, 90, 773-777.

33. Rakha, A.; Saulnier, L.; Åmana, P.; Andersson, R. Enzymatic fingerprinting of arabinoxylan and B-glucan in triticale, barley and tritordeum grains. Carbohyd. Polym. 2012, 90, 1226-1234. [CrossRef]

34. Torres-Pitarch, A.; Manzanilla, E.G.; Gardiner, G.E.; O’Doherty, J.V.; Lawlor, P.G. Systematic review and meta-analysis of the effect of feed enzymes on growth and nutrient digestibility in grow-finisher pigs: Effect of enzyme type and cereal source. Anim. Feed Sci. Technol. 2019, 251, 153-165. [CrossRef]

35. Fabijańska, M.; Bekta, M. The use of naked oat instead of barley in complete feed for fatteners. J. Anim. Feed Sci. 2001, 10, 219-224. [CrossRef]

36. Kirchgessner, M.; Roth, F.X. Schätzgleichungen zur ermittlung des energetischen futterwertes von mischfuttermitteln für schweine. J. Anim. Physiol. Anim. Nutr. 1983, 50, 270-275. [CrossRef]

37. Grela, E.R.; Skomiał, J. (Eds.) Feeding Recommendations and the Nutritional Value of Feeds for Pigs. Standards of Pig Nutrition; The Kielanowski Institute of Animal Physiology and Nutrition Polish Academy of Sciences: Jabłonna, Poland, 2015.

38. Różycki, M. Zasady Postępowania Przy Ocenie Świń w Stacjach Kontroli Użytkowości Rzeźnej Trzody Chlewnej; Stan Hodowli i Wyniki Oceny Świń; National Research Institute of Animal Production: Krakow, Poland, 1996; Volume XIV, pp. 69-82.

39. AOAC. Official Methods of Analysis of the Association of Official Analytical Chemists, 15th ed.; Association of Official Analytical Chemists, Inc.: Washington, DC, USA, 1990; Chapter 32.

40. Mccleary, B.V.; Codd, R. Measurement of (1-3) (1-4)-beta-D-glucan in barley and oats: A streamlined enzymic procedure. J. Sci. Food Agric. 1991, 55, 303-312. [CrossRef]

41. Folch, J.; Lees, M.; Sloane Stanley, G.H. A simple method for the isolation and purification of total lipids from animal tissues. J. Biol. Chem. 1957, 226, 497-509. [CrossRef]

42. Grau, R.; Hamm, R. Eine einfache Methode zur Bestimmung der Wasserbindung im Muskel. Naturwissenschaften 1953, 40, 29-30. [CrossRef]

43. Pohja, M.S.; Ninivaara, F.P. Die Bestimmung der Wasserbindung des Fleisches mittels der Konstantdrück-Methode. Fleischwirtschaft 1957, 9, 193-195.

44. CIE. Draft Standard 014-4.3/E: Colorimetry_Part. 4: CIE 1976 L $^{*} a^{*} b^{*}$ Colour Space; CIE: Vienna, Austria, $2007 ;$ p. 8.

45. Honikel, K.O. Reference methods for the assessment of physical characteristics of meat. Meat Sci. 1998, 49, 447-457. [CrossRef]

46. Milczarek, A.; Osek, M. Effectiveness evaluation of use of various protein feeds for broiler chicken feeding. Ann. Anim. Sci. 2019, 19, 1063-1081. [CrossRef]

47. StatSoft Inc. Statistica (Data Analysis Software System), version 13.1; StatSoft Inc.: Tulsa, OK, USA, 2019.

48. Kosieradzka, M.; Fabijańska, I. Polish naked oats in the intensive pig fattening. In Proceedings of the XXVIIIth Session of Animal Nutrition, Committee on Animal Sciences, Polish Academy of Sciences: "Nutritional Needs of High-Productive Farm Animals", Krynica, Poland, 8-10 September 1999; pp. 259-262.

49. Semeniuk, W.; Grela, E.R. Efektywność żywienia tuczników mieszankami z udziałem owsa nagiego. In Proceedings of the XXXIst Session of Animal Nutrition, Committee on Animal Sciences, Polish Academy of Sciences: “Fizjologiczne Podstawy Żywienia Zwierzat i ich Praktyczne Implikacje", Wrocław, Poland, 11-12 June 2002; p. 100.

50. Stasiak, A.; Kamyk, P.; Lechowski, J. Evaluation of fattening and slaughter traits of Pulawska, PLW and Duroc hybrids. Ann. UMCS Sec. EE Zootech. 2003, 21, 307-311.

51. POLSUS. Available online: https://www.polsus.pl/index.php/en/hodowla/rasy/pulawska (accessed on 15 September 2021).

52. Sieczkowska, H.; Koćwin-Podsiadła, M.; Krzęcio, E.; Antosik, K.; Zybert, A. Quality and technological properties of meat from landrace, Yorkshire Duroc and Landrace-Yorkshire Duroc-Pietrain fatteners. Pol. J. Food Nutr. Sci. 2009, 59, $329-333$.

53. Stanišić, N.; Parunović, N.; Stajić, S.; Petrović, M.; Radović, Č.; Živković, D.; Petričević, M. Differences in meat colour between free-range Swallow Belly Mangalitsa and commercially reared Swedish Landrace pigs during 6 days of vacuum storage. Arch. Anim. Breed. 2016, 59, 159-166. [CrossRef]

54. Florowski, T.; Pisula, A.; Adamczak, L.; Buczyński, J.T.; Orzechowska, B. Technological parameters of meat in pigs of two Polish local breeds Zlotnicka Spotted and Pulawska. Anim. Sci. Pap. Rep. 2006, 24, 217-224.

55. Wajda, S.; Daszkiewicz, T.; Winiarski, R.; Borzuta, K. Correlations between intramuscular fat and tissue content of pig carcasses. Rocz. Instyt. Przem Mięs. Tłuszcz 2004, 41, 119-129.

56. Łyczyński, A.; Pospiech, E.; Koćwin-Podsiadła, M.; Rzosińska, E.; Czyżak-Runowska, G.; Grześ, B.; Krzęcio, E. Level of intramuscular fat selected slaughter traits and pork meat quality. Ann. Anim. Sci. 2004, 2, 235-239. 
57. Grześkowiak, E.; Borys, A.; Borzuta, K.; Buczyński, J.T.; Lisiak, D. Slaughter value, meat quality and back fat fatty acid profile in Zlotnicka Spotted fatteners. Anim. Sci. Pap. Rep. 2009, 27, 115-125.

58. Schwörer, D.; Hofer, A.; Lorenz, D.; Rebsamen, A. Selection progress of intramuscular fat in Swiss pig production. In Proceedings of the Joint Session of the EAAP Commissions on Pig Production, Animal Genetics and Animal Nutrition, Zurich, Switzerland, 25 August 1999; pp. 69-72.

59. Czarniecka-Skubina, E.; Przybylski, W.; Jaworska, D.; Wachowicz, I.; Urbańska, I.; Niemyjski, S. Quality profile pork meat with varying contents of intramuscular fat. Żywność 2007, 6, 285-294.

60. Furman, M.; Malovrh, Š.; Levart, A.; Kovač, M. Fatty acid composition of meat and adipose tissue from Krškopolje pigs and commercial fatteners in Slovenia. Arch. Tierz. 2010, 53, 73-84.

61. Nuernberg, K.; Nuernberg, G.; Priepke, A.; Dannenberger, D. Sea buckthorn pomace supplementation in the finishing diets of pigs-Are there effects on meat quality and muscle fatty acids? Arch. Anim. Breed. 2015, 58, 107-113. [CrossRef]

62. Cebulska, A.; Václavková, E.; Bocian, M.; Dybała, J.; Wiśniewska, J.; Kapelański, W. Quality and dietary value of pork meat of the Puławska and Złotnicka Spotted breeds, and commercial fattening pigs. Ann. Anim. Sci. 2018, 18, 281-291. [CrossRef]

63. Migdał, W.; Zadora, A.; Kozioł, A.; Nowak, J.; Orzechowska, B.; Tyra, M.; Wojtasik, D.; Pustkowiak, H. Fatty acid profile of loin ham from Polish Landrace fatteners with different growth rates. Anim. Sci. 2006, 1, 92-93. 